Pacific Journal of Mathematics

NONTANGENTIAL HOMOTOPY EQUIVALENCES 


\title{
NONTANGENTIAL HOMOTOPY EQUIVALENCES
}

\author{
VICTOR A. BELFI
}

The purpose of this paper is to apply surgery techniques in a simple, geometric way to construct manifolds which are nontangentially homotopy equivalent to certain $\pi$-manifolds. Applying this construction to an $H$-manifold of the appropriate type yields an infinite collection of mutually nonhomeomorphic $H$-manifolds, all nontangentially homotopy equivalent to the given one.

The theorem proved is the following: If $N^{4 k}$ is a smooth, closed, orientable $\pi$-manifold and $L^{m}$ is a smooth, closed, simply connected $\pi$-manifold, there is a countable collection of smooth, closed manifolds $\left\{M_{i}\right\}$ satisfying (1) no $M_{i}$ is a $\pi$ manifold, (2) each $M_{i}$ is homotopy equivalent but not homeomorphic to $N \times L,(3) M_{\imath}$ is not homeomorphic to $M_{j}$ if $i \neq j$.

1. Construction of the surgery problem. In [2] Milnor describes a $(2 k-1)$-connected, bounded $\pi$-manifold of dimension $4 k$ and Hirzebruch index $8(k \geqq 2)$. This manifold, which we denote by $Y^{4 k}$, is obtained by plumbing together 8 copies of the tangent disk bundle of $S^{2 k}$ according to a certain scheme. This implies that $Y$ has the homotopy type of a bouquent of eight $2 k$-spheres. The only other property of $Y$ which we shall need is that $\partial Y$ is a homotopy sphere. Let $r$ be the order of $\partial Y^{4 k}$ in the group of homotopy spheres $b P_{4 k}$ [3] and take $W^{4 / 0}$ to be the $r$-fold connected sum along the boundary of $Y^{4 k}$. By the choice of $r, \partial W$ is diffeomorphic to $S^{4 k-1}$. Attaching a $4 k$-disk to $W$ by a diffeomorphism along the boundary, we obtain a closed, smooth manifold $\hat{W}$, which is $(2 k-1)$-connected and has index 8r. By the Hirzebruch index theorem $\hat{W}$ is not a $\pi$-manifold, but is almost parallelizable.

Define $f: W^{4 k} \rightarrow D^{4 k}$ by the identity on the boundary, stretching a collar of $\partial W$ over $D^{4 k}$, and sending the remainder of $W$ to a point. This gives a degree 1 map $f:(W, \partial W) \rightarrow\left(D^{4 k}, \partial D^{4 k}\right)$ which is tangential since both $W$ and $D^{4 k}$ are $\pi$-manifolds. $f$ is already a homotopy equivalence on the boundary, so we have a surgery problem in the bounded case. The connectedness of $W$ implies that $f$ is already an isomorphism in homology below the middle dimension. However the kernel of $f_{*}$ in dimension $2 k$ is $\frac{\mathrm{z} \oplus \cdots \oplus Z}{8 r}$ and the index of the kernel is the index of $W$ which is $8 r$. Thus it is not possible to complete the surgery.

But if $L^{m}$ is a closed, smooth, simply connected $\pi$-manifold, the surgery problem $f \times 1_{L}: W \times L \rightarrow D^{4 k} \times L$ does have a solution. To 
see this note first that if $m$ is odd, the problem is odd dimensional so there are no obstructions to modifying $W \times L$ and $f \times 1_{L}$ by surgery to obtain a homotopy equivalence. If $m \equiv 0(\bmod 4)$, the problem has an index obstruction given by the product of the index obstruction of the map $f$ and the index of the manifold $L$, i.e., $I\left(f \times 1_{L}\right)=$ $I(f) \cdot I(L)$. This product vanishes since $L$ is a $\pi$-manifold. The formula follows from the multiplicativity of the index of a manifold. If $m \equiv 2(\bmod 4)$ the problem has a Kervaire invariant obstruction given by the mod 2 product of the Kervaire invariant obstruction of $f$ and the Euler characteristic of $L$, the formula arising from Sullivan's characterization of the Kervaire invariant obstruction [8]. Since $L$ is a $\pi$-manifold, $\chi(L)=0$; so $K\left(f \times 1_{L}\right)$ vanishes as well.

Now we change the surgery problem discussed above into a problem for closed manifolds. Let $N$ be a smooth, closed, $\pi$-manifold of dimension $4 k$. Take a small disk $D^{4 k}$ in $N$ and form the connected sum $N \# \hat{W}$ using this disk and the disk attached to $W$ to make $\hat{W}$. Define $1_{N} \# f: N \# \hat{W} \rightarrow N$ by the identity on $N$-int $D^{4 k}$ and $f$ on $W$. Although $\left(1_{N} \# f\right) \times 1_{L}$ is not tangential, it can be surgered to a homotopy equivalence. This is because it is already a homotopy equivalence except on $W \times L$, where it is tangential; so it suffices to do surgery on $W \times L$ leaving the boundary fixed to make $N \# \hat{W} \times L$ homotopy equivalent to $N \times L$. We have already seen that this can be done. Summing up the discussion we have

Proposition 1. Suppose $N^{4 k}$ is a closed, smooth, orientable $\pi$ manifold and $L^{m}$ is a closed, smooth, simply connected $\pi$-manifold. Then there is a manifold $M^{4 k+m}$, homotopy equivalent to $N \times L$ obtained by surgery on $\left(1_{N} \# f\right) \times 1_{L}$.

Notice that if $W_{i}^{4 k}=\underbrace{W^{4 k} \# \cdots \# W^{4 k}}_{i}$, and we define $f_{i}: W_{i} \rightarrow D^{4 k}$ the same way as we defined $f$, the above considerations also apply to $W_{i}$. The only difference is that $W_{i}$ has index $8 \mathrm{ri}$. We shall denote the solution to the surgery problem using $W_{i}$ by $M_{i}^{4 k+m}$.

We also remark here that $M$, as a solution to a given surgery problem, is unique up to $P L$ homeomorphism, but not not always up to diffeomorphism. This follows from Novikov's results [5]. Since we shall be primarily concerned with the topological type of such solutions, we shall ignore this ambiguity.

\section{Properties of the surgery solution.}

Proposition 2. The manifold $M^{4 k+m}$ obtained by surgery on 


$$
\left(1_{N} \# f\right) \times 1_{L}: N \# \hat{W} \times L \rightarrow N \times L
$$

is not a $\pi$-manifold.

Proof. After surgery we have a homotopy equivalence $g: M \rightarrow$ $N \times L$ and a cobordism $Z$ between $M$ and $N \# W \times L$ together with a map $F: Z \rightarrow N \times L$ whose restriction is $g$ on $M$ and $\left(1_{N} \sharp f\right) \times 1_{L}$ on $N \# \hat{W} \times L$. If $*$ is a point of $L,\left(1_{N} \# f\right) \times 1_{L}$ is transverse regular with respect to $N \times^{*}$. Change $g$ by a small homotopy to make it transverse regular with respect to $N \times *$. Finally leaving $\left(1_{N} \# f\right) \times$ $1_{L}$ and $g$ fixed, make $F$ transverse regular with respect to $N \times{ }^{*}$ to obtain the oriented cobordism $F^{-1}\left(N \times{ }^{*}\right)$ between $N \# \hat{W}$ and

$$
S=g^{-1}\left(N \times{ }^{*}\right) \text {. }
$$

Because $N \# \hat{W}$ and $S$ are oriented cobordant, $I(S)=I(N \# \hat{W}) \neq 0$. We have the usual equivalence of tangent and normal bundles

$$
\tau(M) \mid S \cong \tau(S) \oplus \nu(S \subset M) .
$$

Since $f$ is transverse regular with respect to $N \times{ }^{*}$ and

$$
\nu(N \times * \subset N \times L)
$$

is trivial, $\nu(S \subset M)$ is trivial. Thus if $\nu(M) \mid S$ were stably trivial, $\tau(S)$ would be stably trivial, contradicting $I(S) \neq 0$. Therefore $\tau(M) \mid S$ is not stably trivial and consequently $\tau(M)$ is not stably trivial.

Proposition 3. $M$ is not homeomorphic to $N \times L$.

Proof. Suppose $h: M \rightarrow N \times L$ is a homeomorphism. Denote by $p_{j}(M)$ the $j^{t h}$ Pontrjagin class of $M$ (i.e., of $\tau(M)$ ) and by $p_{j}(M ; \mathbf{Q})$ the $j^{t h}$ rational Pontrjagin class of $M$. In the proof of Proposition 2 it was shown that $M^{4 k+m}$ contains a closed submanifold $S$ of dimension $4 k$ and index $8 r$. If $i: S \rightarrow M$ is inclusion, the Hirzebruch index theorem implies

$$
\begin{aligned}
8 r & =\left\langle L_{k}\left(p_{1}(S), \cdots, p_{k}(S)\right),[S]\right\rangle \\
& =\left\langle L_{k}\left(i^{*} p_{1}(M), \cdots, i^{*} p_{k}(M)\right),[S]\right\rangle \\
& =\left\langle L_{k}\left(p_{1}(M), \cdots, p_{k}(M)\right), i_{*}[S]\right\rangle .
\end{aligned}
$$

Now we may replace $p_{j}(M)$ by $p_{j}(M ; \mathbf{Q})$ since any torsion evaluated on the orientation class is zero. By the topological invariance of rational Pontrjagin classes, $p_{j}(M ; \mathbf{Q})=h^{*}\left(p_{j}(N \times L)\right.$; $\left.\mathbf{Q}\right)$; but

$$
p_{j}(N \times L ; \mathbf{Q})=0
$$

for every $j$ because $N \times L$ is a $\pi$-manifold. Therefore $p_{j}(M ; \mathbf{Q})=0$ 
for every $j$, a contradiction.

Observe that Propositions 2 and 3 are likewise valid for the manifolds $M_{\imath}$, each $M_{\imath}$ containing a closed submanifold $S_{i}$ of dimension $4 k$ and index $8 \mathrm{ri}$.

Now we are in a position to prove the central theorem of this paper.

THEOREM 1. Suppose $N$ is a smooth, closed, orientable $\pi$-manifold of dimersion $4 k(k \geqq 2)$ and $L$ is a smooth, closed simply connected $\pi$ manifold. Then there is a countable sequence of smooth, closed manifolds $\left\{M_{\imath}\right\}$ having the following properties: (1) no $M_{i}$ is a $\pi$-manifold, (2) each $M_{i}$ is homotopy equivalent but not homeomorphic to $N \times L$, (3) $M_{i}$ is not homeomorphic to $M_{3}$ if $i \neq j$.

Proof. The $M_{i}$ 's are the surgery solutions already described. Propositions 2 and 3 establish (1) and (2). It remains to prove (3). We do this by expanding the idea of the proof of Proposition 3.

Suppose there exists a homeomorphism $h: M_{\jmath} \rightarrow M_{\imath}$ and $i \neq j$, say $i>j$. (For the rest of this paragraph $t=i, j$.) Let $g_{i}: M_{t} \rightarrow N \times L$ be a homotopy equivalence which is transverse regular with respect to $N \times *$ so that $g_{t}^{-1}(N \times *)=S_{t}$ where $I\left(S_{t}\right)=8 r t$. (We may assume that $g_{t}$ is still the identity on $\left(N-\operatorname{int} D^{4 k}\right) \times L$ since no surgery is done there.) Then by the index theorem,

$$
\left\langle L_{k}\left(p_{I}\left(M_{t} ; \mathbf{Q}\right), \cdots, p_{k}\left(M_{t} ; \mathbf{Q}\right)\right),\left[S_{t}\right]\right\rangle=I\left(S_{t}\right) .
$$

To simplify notation we omit explicit reference to the inclusion maps $S_{t} \subset M_{t}$ and abbreviate $L_{k}\left(p_{1}(X ; \mathbf{Q}), \cdots, p_{k}(X ; \mathbf{Q})\right)$ by $L_{k}(X)$. Let $\bar{g}_{t}$ be a homotopy inverse for $g_{t}$. The idea is then to show that $g_{i} h \bar{g}_{j}$ does not behave properly on rational homology. We shall be referring to the following diagram for the rest of the proof:

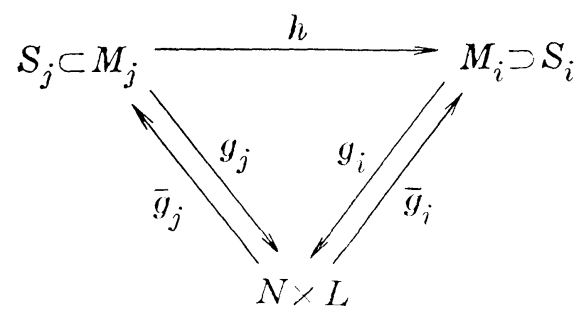

By the transverse regularity of $g_{t}$, it follows that

$$
g_{t_{*}}\left[S_{t}\right]=\left[N \times{ }^{*}\right]=[N] \otimes 1 \in H_{4 k}(N \times L ; \mathbf{Q}),
$$

so $g_{J_{*}} \bar{g}_{i_{*}}\left[S_{i}\right]=\left[S_{\jmath}\right]$. Thus 


$$
I\left(S_{j}\right)=\left\langle L_{k}\left(M_{j}\right), \bar{g}_{J_{*}} g_{i_{*}}\left[S_{i}\right]\right\rangle=\left\langle L_{k}\left(M_{i}\right), h_{*} \bar{g}_{j_{*}} g_{i_{*}}\left[S_{i}\right]\right\rangle
$$

by the topological invariance of rational Pontrjagin classes.

Define a bundle $\xi$ over $N \times L$ by $\bar{g}_{i}^{*}\left(\tau\left(M_{i}\right)\right)$. This means that $\tau\left(M_{i}\right)=g_{i}^{*}(\xi)$. Since $g_{i}$ is the identity on $N-\operatorname{int} D^{4 k} \times L$ and

$$
\tau\left(M_{i}\right) \mid N-\operatorname{int} D^{4 k} \times L
$$

is trivial, it follows that $\xi \mid N-\operatorname{int} D^{4 k} \times L$ is trivial. Now if

$$
i: N-\operatorname{int} D^{4 k} \times L \rightarrow N \times L
$$

is inclusion, then if $x \otimes y \in H_{*}(N \times L$; Q $)$ and $\operatorname{dim} x<4 k, x \otimes y \in$ image $i_{*}$, say $x \otimes y=i_{*} z$. Thus $\left\langle L_{k}(\xi), x \otimes y\right\rangle=\left\langle L_{k}\left(i^{*} \xi\right), z\right\rangle=0$ since $i^{*} \xi$ is trivial. This shows that if $\gamma_{4 k} \in H_{4 k}(N \times L ; \mathbf{Q})$, then $\left\langle L_{k}(\xi), \gamma_{4 k}\right\rangle$ is given by the product of the coefficient of $[N] \otimes 1$ in $\gamma_{4 k}$ and

$$
\left\langle L_{k}(\xi),[N] \otimes 1\right\rangle \text {. }
$$

Using the preceding observation, we can compute the coefficient of $[N] \otimes 1$ in $\left(g_{2} h \bar{g}_{\imath}\right)_{*}[N] \otimes 1$ as follows.

$$
\begin{aligned}
\left\langle L_{k}(\xi),\left(g_{i} h \bar{g}_{j}\right)_{*}[N] \otimes 1\right\rangle & =\left\langle L_{k}\left(M_{i}\right), h_{*} \bar{g}_{j_{*}}[N] \otimes 1\right\rangle \\
& =\left\langle L_{k}\left(M_{i}\right), h_{*} \bar{g}_{j_{*}} g_{i_{*}}\left[S_{i}\right]\right\rangle \\
& =I\left(S_{j}\right)=(j / i) I\left(S_{i}\right) .
\end{aligned}
$$

But

$$
I\left(S_{i}\right)=\left\langle L_{k}\left(M_{i}\right),\left[S_{i}\right]\right\rangle=\left\langle L_{k}(\xi), g_{i_{*}}\left[S_{i}\right]\right\rangle=\left\langle L_{k}(\xi),[N] \otimes 1\right\rangle .
$$

Hence this coefficient is $j / i$ which is not an integer since $i>j$. This contradicts the fact that any induced map on rational homology must send integral classes to integral classes.

3. An extension of the results. It has been pointed out to me that the results of this paper can be extended in the following way:

If $M^{n}$ is a simply connected smooth manifold where $n$ is odd and $H^{4 k}(M ; \mathbf{Q}) \neq 0$ or some $4 k<n$, the Pontrjagin character shows that $\widetilde{K O}(M)$ is infinite. (See, for example, Hsiang [2].) Thus the kernel of $\widetilde{K O}(M) \rightarrow J(M)$ is infinite. It can be shown that the result of doing surgery on the elements of the kernel is a collection of smooth manifolds homotopy equivalent to $M$ containing an infinite subset $\left\{M_{i}\right\}$ of mutually non-homeomorphic manifolds. The condition on the rational cohomology of $M$ is also necessary for the manifolds $\left\{M_{2}\right\}$ exist.

Although the theorem described above considerably extends the class of manifolds to which the principal result applies, its proof requires methods of a deeper sort and the geometric simplicity is lost. 
4. Applications. By an $H$-manifold we mean a closed, orientable topological manifold having the structure of an $H$-space.

TheOREm 2. Suppose $N^{4 k}$ and $L^{m}$ are smooth $H$-manifolds, $N$ and $L$ are $\pi$-manifolds, and $L$ is simply connected. Then there exists a sequence of mutually nonhomeomorphic smooth $H$-manifolds $\left\{M_{i}\right\}$ satisfying (1) no $M_{i}$ is a $\pi$-maifold, (2) each $M_{i}$ is homotopy equivalent, but not homeomorphic to $N \times L$.

Proof. This is immediate from Theorem 1 since the product of $2 \mathrm{H}$-manifolds is an $\mathrm{H}$-manifold and any manifold homotopy equivalent to an $H$-manifold is itself an $H$-manifold.

Examples of manifolds nontangentially homotopy equivalent to Lie groups were known before surgery techniques were introduced; however all these were nonsimply connected. An example due to Milnor of a manifold homotopy equivalent to $S^{1} \times S^{3} \times S^{7}$ with a nonzero Pontrjagin class is quoted by Browder and Spanier [1].

The recent results of a A. Zabrodsky [9] and J. Stasheff [7] have produced new homotopy types of $H$-manifolds (other than compact Lie groups) to which Theorem 2 applies. However if we restrict ourselves to simply connected, compact Lie groups, we can obtain a stronger conclusion.

THEOREM 3. Suppose $N^{4 k}$ and $L^{m}$ are simply connected compact Lie groups $(k \geqq 2)$. Then there is a countable sequence of mutually nonhomeomorphic $H$-manifolds $\left\{M_{i}\right\}$ satisfying (1) no $M_{i}$ is a $\pi$-manifold, (2) each $M_{i}$ is homotopy equivalent to $N \times L$ but not homeomorphic to any Lie group.

Proof: Since Lie groups are $\pi$-manifolds, Theorem 1 applies. H. Scheerer has proved [6] that homotopy equivalent, compact, simply connected Lie groups are isomorphic; so if $M_{i}$ were homeomorphic to any Lie group, it would be homeomorphic to $N \times L$, contradicting Theorem 1.

The author is indebted to John W. Morgan for his invaluable suggestions in the course of this research and to the refree for pointing out related results.

\section{REFERENCES}

1. W. Browder and E. Spanier, H-spaces and duality, Pacific J. Math. 12 (1962), 411414 .

2. W.-C. Hsiang, A note on free differentiable actions of $S^{1}$ and $S^{3}$ on homotopy spheres, Ann. of Math. 83 (1966), 266-272. 
3. M. A. Kervaire and J. W. Milnor, Groups of homotopy spheres: I, Ann. of Math. 77 (1963), 504-537.

4. J. W. Milnor, "Differential topology," Lectures on Modern Mathematics, Vol. II, T. L. Saaty, ed., John Wiley and Sons, Inc., New York, 1964.

5. S. P. Novikov, Homotopically equivalent smooth manifolds, I, Trans. Amer. Math. Soc. (2) 48 (1965), 271-396.

6. H. Scheerer, Homotopieaquivalente kompacte Liesche Gruppen, Topology 7 (1968), $227-232$.

7. J. Stasheff, Manifolds of the homotopy type of (non-Lie) groups, Bull. Amer. Math. Soc. 75 (1969), 998-1000.

8. D. Sullivan, Geometric Topology Seminar Notes Mimeographed, Princeton University. 9. A. Zabrodsky, Homotopy associativity and finite $C W$ complexes, Mimeographed Notes, University of Illinois, Chicago Circle, Ill., 1968.

Received October 3, 1969, and in revised form June 9, 1970. The author held a NASA graduate fellowship while this research was done for his $\mathrm{Ph}$. $\mathrm{D}$. thesis at Rice University in 1969.

TEXas Christian UNIVERSITY 



\title{
PACIFIC JOURNAL OF MATHEMATICS
}

\section{EDITORS}

H. SAMELSON

Stanford University

Stanford, California 94305

C. R. HobвY

University of Washington

Seattle, Washington 98105

\section{J. DugunduI}

Department of Mathematics

University of Southern California

Los Angeles, California 90007

RICHARD ARENS

University of California

Los Angeles, California 90024

\section{ASSOCIATE EDITORS}
E. F. BECKENBACH
B. H. Neumann
F. WOLE
K. Yoshida

\section{SUPPORTING INSTITUTIONS}

\author{
UNIVERSITY OF BRITISH COLUMBIA \\ CALIFORNIA INSTITUTE OF TECHNOLOGY \\ UNIVERSITY OF CALIFORNIA \\ MONTANA STATE UNIVERSITY \\ UNIVERSITY OF NEVADA \\ NEW MEXICO STATE UNIVERSITY \\ OREGON STATE UNIVERSITY \\ UNIVERSITY OF OREGON \\ OSAKA UNIVERSITY \\ UNIVERSITY OF SOUTHERN CALIFORNIA
}

\author{
STANFORD UNIVERSITY \\ UNIVERSITY OF TOKYO \\ UNIVERSITY OF UTAH \\ WASHINGTON STATE UNIVERSITY \\ UNIVERSITY OF WASHINGTON

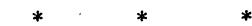 \\ AMERICAN MATHEMATICAL SOCIETY \\ CHEVRON RESEARCH CORPORATION \\ NAVAL WEAPONS CENTER
}

The Supporting Institutions listed above contribute to the cost of publication of this Journal, but they are not owners or publishers and have no responsibility for its content or policies.

Mathematical papers intended for publication in the Pacific Journal of Mathematics should be in typed form or offset-reproduced, (not dittoed), double spaced with large margins. Underline Greek letters in red, German in green, and script in blue. The first paragraph or two must be capable of being used separately as a synopsis of the entire paper. The editorial "we" must not be used in the synopsis, and items of the bibliography should not be cited there unless absolutely necessary, in which case they must be identified by author and Journal, rather than by item number. Manuscripts, in duplicate if possible, may be sent to any one of the four editors. Please classify according to the scheme of Math. Rev. Index to Vol. 39. All other communications to the editors should be addressed to the managing editor, Richard Arens, University of California, Los Angeles, California, 90024.

50 reprints are provided free for each article; additional copies may be obtained at cost in multiples of 50 .

The Pacific Journal of Mathematics is published monthly. Effective with Volume 16 the price per volume (3 numbers) is $\$ 8.00$; single issues, $\$ 3.00$. Special price for current issues to individual faculty members of supporting institutions and to individual members of the American Mathematical Society: $\$ 4.00$ per volume; single issues $\$ 1.50$. Back numbers are available.

Subscriptions, orders for back numbers, and changes of address should be sent to Pacific Journal of Mathematics, 103 Highland Boulevard, Berkeley, California, 94708.

PUBLISHED BY PACIFIC JOURNAL OF MATHEMATICS, A NON-PROFIT CORPORATION

Printed at Kokusai Bunken Insatsusha (International Academic Printing Co., Ltd.), 7-17, Fujimi 2-chome, Chiyoda-ku, Tokyo, Japan. 


\section{Pacific Journal of Mathematics}

\section{Vol. 36, No. $3 \quad$ BadMonth, 1971}

E. M. Alfsen and B. Hirsberg, On dominated extensions in linear subspaces of

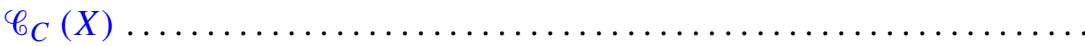

Joby Milo Anthony, Topologies for quotient fields of commutative integral

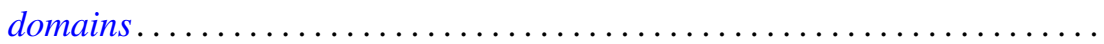

V. Balakrishnan, G. Sankaranarayanan and C. Suyambulingom, Ordered cycle lengths in a random permutation .......................... 603

Victor Allen Belfi, Nontangential homotopy equivalences............... 615

Jane Maxwell Day, Compact semigroups with square roots .............. 623

Norman Henry Eggert, Jr., Quasi regular groups of finite commutative nilpotent algebras .......................................... 631

Paul Erdős and Ernst Gabor Straus, Some number theoretic results ......... 635

George Rudolph Gordh, Jr., Monotone decompositions of irreducible Hausdorff continua .............................................

Darald Joe Hartfiel, The matrix equation $A X B=X \ldots \ldots \ldots \ldots \ldots \ldots . \ldots 69$

James Howard Hedlund, Expansive automorphisms of Banach spaces. II . . . . 671

I. Martin (Irving) Isaacs, The p-parts of character degrees in p-solvable

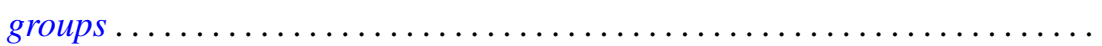

Donald Glen Johnson, Rings of quotients of $\Phi$-algebras ............... 693

Norman Lloyd Johnson, Transition planes constructed from semifield planes....

Anne Bramble Searle Koehler, Quasi-projective and quasi-injective modules.

James J. Kuzmanovich, Completions of Dedekind prime rings as second endomorphism rings...

B. T. Y. Kwee, On generalized translated quasi-Cesàro summability ...

Yves A. Lequain, Differential simplicity and complete integral closure

741

Mordechai Lewin, On nonnegative matrices.

753

Kevin Mor McCrimmon, Speciality of quadratic Jordan algebras ...

Hussain Sayid Nur, Singular perturbations of differential equations in abstract spaces .....................................

D. K. Oates, A non-compact Krein-Milman theorem .

Lavon Barry Page, Operators that commute with a unilateral shift on an invariant subspace...

Helga Schirmer, Properties of fixed point sets on dendrites.

Saharon Shelah, On the number of non-almost isomorphic models of $T$ in a

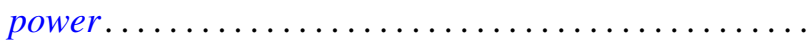

Robert Moffatt Stephenson Jr., Minimal first countable Hausdorff spaces....

Masamichi Takesaki, The quotient algebra of a finite von Neumann algebra 